\title{
WSRT observations and surface photometry of two unusual spiral galaxies ${ }^{\star}$
}

\author{
A. S. Saburova ${ }^{1}$, G. I. G. Józsa ${ }^{2,3}$, A. V. Zasov ${ }^{1}$, and D. V. Bizyaev ${ }^{4,1}$ \\ ${ }^{1}$ Moscow M.V. Lomonosov State University, Sternberg Astronomical Institute, Universitetskii pr. 13, 119992 Moscow, Russia \\ e-mail: saburovaann@gmail.com \\ 2 Netherlands Institute for Radio Astronomy, Postbus 2, 7990 AA Dwingeloo, The Netherlands \\ e-mail: jozsa@astron.nl \\ 3 Argelander - Institut für Astronomie, Auf dem Hügel 71, 53121 Bonn, Germany \\ ${ }^{4}$ New Mexico State University and Apache Point Observatory, Sunspot, NM 88349, USA \\ e-mail: dmbiz@apo.nmsu.edu
}

Received 13 December 2012 / Accepted 30 April 2013

\section{ABSTRACT}

\begin{abstract}
We discuss the results of a mass decomposition of two spiral galaxies, NGC 6824 and UGC 11919. In a previous analysis of the Hyperleda catalog, the galaxies were identified as having a peculiar dynamical mass-to-light ratio. The aim of this study is to confirm or disprove the preliminary findings, indicating a non-standard stellar initial mass function (IMF) for the galaxies. The surface photometry in $B, V$, and $R$ bands was carried out with the Apache Point $0.5-\mathrm{m}$ telescope and the H I data cubes were obtained with the Westerbork Synthesis Radio Telescope (WSRT). Photometric profiles were decomposed into bulge and exponential disk components. Using the obtained H I data cubes, rotation curves of both galaxies were constructed. Employing the photometric profiles, the mass distribution of the galaxies was decomposed into mass components: bulge, stellar disk, gas, and pseudo-isothermal dark halo. We conclude that NGC 6824 possesses a stellar disk with mass-to-light ratio $\left(M / L_{B}\right)_{\text {disk }}=2.5$, in agreement with its color $(B-V)_{0}$. On the contrary, UGC 11919 appears to have a very lightweight disk. Its dynamically estimated mass corresponds to a low stellar disk mass-to-light ratio $\left(M / L_{B}\right)_{\text {disk }} \approx 0.5$. Under standard assumptions, this ratio does not agree with the relatively red color of the disk, while a bottom light stellar initial mass function is needed to explain the observations.
\end{abstract}

Key words. galaxies: kinematics and dynamics - galaxies: individual: NGC 6824 - galaxies: structure - galaxies: photometry galaxies: spiral - galaxies: individual: UGC 11919

\section{Introduction}

The total mass-to-light ratio of a galaxy $(\mathrm{M} / \mathrm{L})$ is a key observable comprising information about the galaxy's evolutional status, its stellar initial mass function (IMF), and the relative masses of its stellar disk and dark matter halo. Hence, for a given galaxy, a peculiar $\mathrm{M} / \mathrm{L}$ for the observed color index may indicate both unusual dynamical properties and/or a non-standard stellar IMF.

The universality of the IMF is a matter of hot debate. There is evidence both in favor and against its uniformity among galaxies and galaxy types. The current consensus shared among a number of studies is that the IMF remains the same for different galaxies (see Kroupa 2012, and references therein). This is inferred from star counts in the Milky Way and in nearby galaxies such as the Large Magellanic Cloud (Kroupa 2002; Gilmore 2001; Bastian et al. 2010). However, several authors argue against the universality of the IMF.

Meurer et al. (2009) present a correlation between the ratio of the $\mathrm{H}_{\alpha}$ line and far UV fluxes $F_{\mathrm{H}_{\alpha}} / F_{\mathrm{FUV}}$ (corrected for the extinction) and the surface brightness in $R$-band. This ratio also appears to correlate with the rotation velocities, luminosities, and dynamical masses of galaxies. The observed range of $F_{\mathrm{H}_{\alpha}} / F_{\mathrm{FUV}}$

* FITS files of H I data cubes are available in electronic form at the CDS via anonymous ftp to cdsarc.u-strasbg. fr (130.79.128.5) or via

http://cdsarc.u-strasbg.fr/viz-bin/qcat?]/A+A/554/A128 could be explained in terms of a variation of either the high mass end or the slope of the IMF.

According to a study of $\sim 300$ galaxies with distances lower than $11 \mathrm{Mpc}$ and star formation rates (SFRs) spanning an interval of five orders, the ratio $F_{\mathrm{H}_{\alpha}} / F_{\mathrm{FUV}}$ is roughly constant only for normal spirals with a star formation rate of $S F R \sim 1 M_{\odot} /$ year, but for dwarf galaxies $F_{\mathrm{H}_{\alpha}} / F_{\mathrm{FUV}}$ decreases with decreasing luminosity (Lee et al. 2009).

High dynamical $\mathrm{M} / \mathrm{Ls}$ in the $V$-band for ultra-compact dwarf galaxies may also indicate the presence of non-universal IMFs with a large fraction of stellar remnants or low-mass stars (Dabringhausen et al. 2010).

By comparison of equivalent widths of the $\mathrm{H}_{\alpha}$ line for a large sample of galaxies, Hoversten \& Glazebrook (2008) show that the IMF could change systematically with the luminosity of galaxies. Using the $\mathrm{H} \alpha$ line width and the color to confine the IMF, they conclude that galaxies with lower luminosities contain a lower fraction of massive stars. Near-infrared observations of disky galaxies by Zackrisson et al. (2006) show that their stellar halos possess extremely red colors which could be explained by a non-standard stellar IMF with an excess in low mass stars. For a sample of $\sim 33000$ galaxies, Gunawardhana et al. (2011) show that the IMF depends on the SFR: galaxies with higher SFR tend to contain more massive stars. Finally, Goudfrooij \& Kruijssen (2013) find that the optical colors of metal-rich globular clusters associated with seven massive elliptical galaxies are bluer than 
Table 1. Main properties of the observed galaxies based on Hyperleda.

\begin{tabular}{|c|c|c|c|c|c|c|c|}
\hline Galaxy & $\begin{array}{l}\text { Type } \\
\text { (2) }\end{array}$ & $\begin{array}{c}D, \mathrm{Mpc} \\
\text { (3) }\end{array}$ & $\begin{array}{l}i\left(^{\circ}\right) \\
(4)\end{array}$ & $\begin{array}{c}v_{\text {rot }} \\
\left(\mathrm{km} \mathrm{s}^{-1}\right) \\
(5)\end{array}$ & $\begin{array}{c}\text { Flux H I } \\
\left(\mathrm{Jy} \mathrm{km} \mathrm{s}^{-1}\right) \\
(6)\end{array}$ & $\begin{array}{c}(B-V)_{0} \\
(7)\end{array}$ & $\begin{array}{l}b t c \\
\text { (8) }\end{array}$ \\
\hline UGC 11919 & $\mathrm{SABb}$ & 74.3 & 54.8 & 71.6 & 5.78 & 0.74 & 12.89 \\
\hline NGC 6824 & Sab & 50.5 & 46 & 359 & 3.24 & 0.65 & 12.19 \\
\hline
\end{tabular}

Notes. (1) Name; (2) morphological type; (3) accepted distance; (4) inclination angle; (5) rotational velocity; (6) H I total flux; (7) (B - V) 0 color index corrected for the Galactic extinction; (8) apparent $B$-band stellar magnitude corrected for the Galactic extinction.

those of parent galaxies at a given radius. They explain the observed color difference by the presence of a bottom-heavy IMF in the observed galaxies.

The question whether the IMF is universal is highly relevant. Stellar population models, which connect star formation history and stellar mass with photometric or spectrophotometric data, are usually based on the assumption of the universality of the IMF (see, e.g., Bell \& de Jong 2001; Bruzual \& Charlot 2003; Into \& Portinari 2013 and others).

In the following, we identify the standard IMF (and hence the standard IMF-based M/L - color relationship) with the scaled (bottom-light) Salpeter-like IMF (Bell \& de Jong 2001). For evolutionary models with exponentially decaying star formation rates, this IMF leads to a $\mathrm{M} / \mathrm{L}$ - color relationship which is practically identical to the relationship based on the Kroupa' IMF (see Appendix B in Portinari et al. 2004). It is worth mentioning that the Salpeter-light IMF agrees with dynamical mass estimates of stellar populations obtained both from rotation curves (Bell \& de Jong 2001) as well as from measurements of the stellar velocity dispersion of spiral disks with known color indices (Zasov et al. 2011). It is not the aim of the present paper to study the universal IMF applicable for most of spiral galaxies. Instead we try to find outliers, galaxies which may possess a non-typical $\mathrm{M} / \mathrm{L}$ for their color, and to confirm their existence.

Low surface brightness galaxies (LSBs) may be a good example for a possibly peculiar IMF. Based on stellar population models with the standard IMF they are believed to be dark halo dominated systems. However, some independent estimates of their disk masses give evidence that LSB disks may have extremely high disk M/Ls (see, e.g., Saburova 2011; Fuchs 2003), which can be explained if their IMF is bottom-heavy and hence deviates from the standard IMF towards the excess of lowmassive stars (see Lee et al. 2004). Alternatively, their disks may contain some fraction of dark matter in non-baryonic (Read et al. 2009) or baryonic form. We note that whereas the excess of massive stars may be revealed from $\mathrm{H}_{\alpha}$ or UV data, the excess or deficit of low massive stars can be found using mass decompositions only.

In our previous studies we searched for high surfacebrightness disk galaxies that may possess non-standard $\mathrm{M} / \mathrm{Ls}$, either too high or too low for the observed color indices (see Saburova et al. 2009), using catalog data, which might be contaminated by individual errors. Errors in rotation velocity or possibly in conjunction with errors in orientation angle(s), which may be responsible for the overestimation or underestimation of the galaxy's mass cannot be excluded in that study and should be confirmed independently. The other aspect which should be taken into account is the presence of a dark matter halo. Within the optical radial extent of the disk, its contribution to the total mass of a galaxy can be comparable to that of its stellar components (Zasov et al. 2011). This means that M/L of the stellar population should always be lower than the total $\mathrm{M} / \mathrm{L}$ of a galaxy within any radial distance. Hence, if the total $\mathrm{M} / \mathrm{L}$ is too low for the color of a galaxy, including the dark matter (DM) halo makes the stellar IMF even more peculiar. In the opposite case, if the total $\mathrm{M} / \mathrm{L}$ is unusually high for the color index of a galaxy, the situation becomes ambiguous: either the galaxy possesses an extremely massive dark halo within the optical radius, or it possesses a bottom-heavy stellar IMF. A mass decomposition may solve the ambiguity, if some additional information, e.g., the radial scalelength of the disk, is known.

In Saburova et al. (2009), making use of Hyperleda, we estimate total $\mathrm{M} / \mathrm{L}$ ratios within the optical radius $R_{25}$ for a sample of $\sim 1300$ galaxies. The obtained total M/L ratios were correlated with the $(B-V)_{0}$ color indices of the galaxies in our sample. Then we compared the positions of the galaxies on the $\left(M / L_{B}\right)-(B-V)_{0}$ diagram with the relation based on stellar population synthesis modeling by Bell \& de Jong (2001) for the standard IMF. We select the galaxies which significantly deviate from the model relation, because peculiarly high $\mathrm{M} / \mathrm{L}$ ratios (if they are not the result of observational errors) can either be the result of a very heavy disk with bottom heavy IMF (with a lack of massive stars) or reflect an unusually large contribution of dark matter in baryonic or non-baryonic form to the total mass of a galaxy. Peculiarly low dynamical M/L can indicate a non-universal IMF with a deficit of low massive stars.

In this work we present the detailed follow-up observations of the two galaxies NGC 6824 and UGC 11919, which were selected as extreme cases from the parent sample as described above. They were observed in the HI emission line with the Westerbork Synthesis Radio Telescope (WSRT). In addition, multicolor surface photometry with the Apache-Point $0.5 \mathrm{~m}$ telescope was carried out. The data were used to construct a mass model of the galaxies and to estimate the masses of their stellar components. This allows us to check whether the peculiar values of $\mathrm{M} / \mathrm{L}$ of the galaxies can be confirmed. The main properties of the galaxies, taken from the Hyperleda database and the accepted distances from NED are given in Table 1.

\section{Observations and data reduction}

\subsection{Radio observations}

We conducted H I observations of NGC 6824 and UGC 11919 with the WSRT in August and September 2011. The observational parameters are listed in Table 2. We used a bandwidth of $10 \mathrm{MHz}$, subdivided into 1024 channels observed in two parallel-handed polarization products. The linewidth of the observed channels is $2.06 \mathrm{~km} \mathrm{~s}^{-1}$. We performed a standard data reduction with the Miriad software package (Sault et al. 1995).

After flagging bad data and a primary bandpass and gain calibration, a first continuum subtraction was performed using a polynomial spectral fit to the visibilities. To correct the frequency-independent gains, a self-calibration was applied to the resulting continuum data (the fitted continuum model). The 

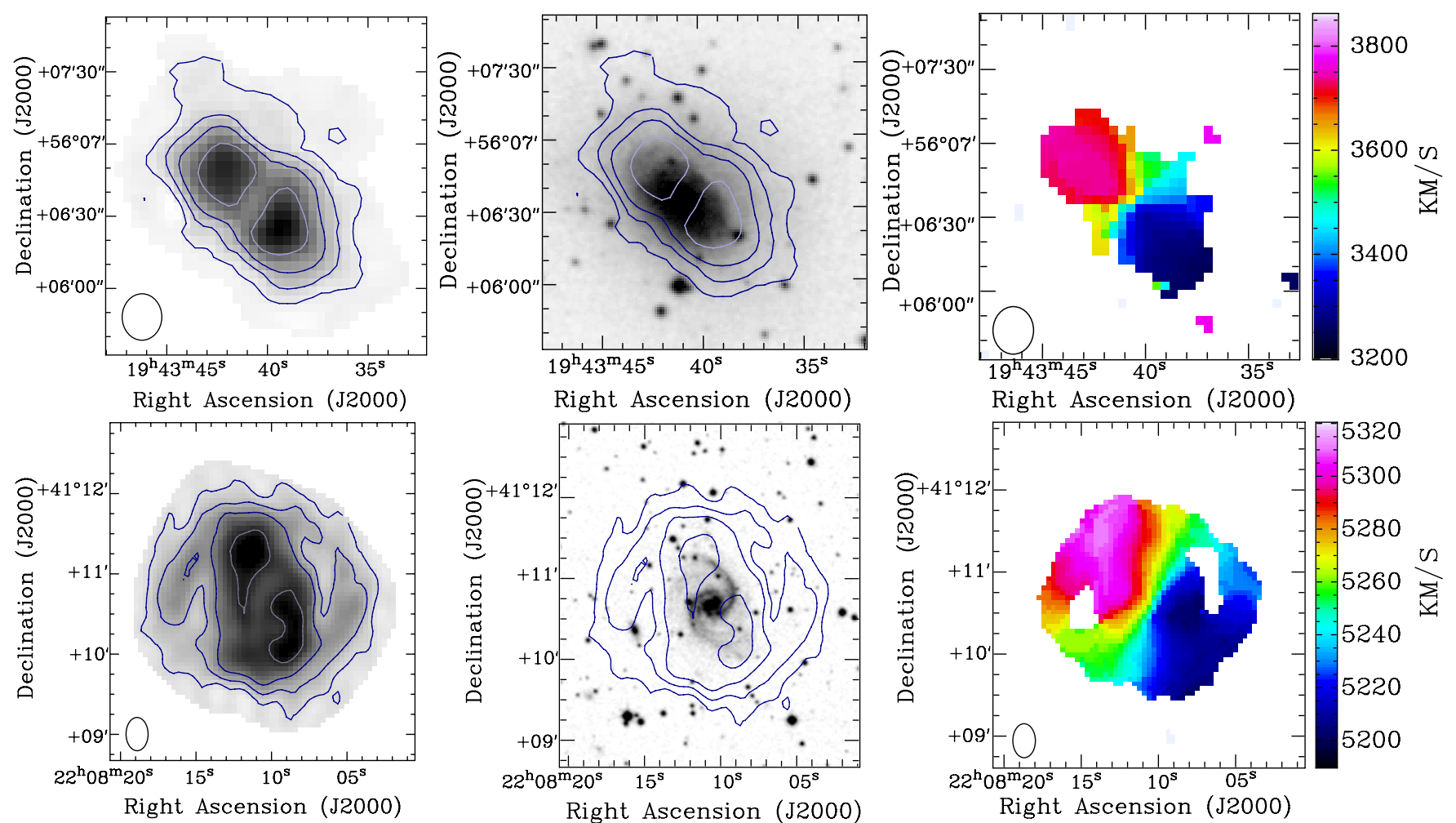

Fig. 1. Top: H I total intensity map and $R$-band optical image of NGC 6824 with overplotted H I total intensity contours: $7.0,26,45,104 \times$ $10^{19}$ atoms $\mathrm{cm}^{-2}$ (left and middle panels respectively) and first-moment map of NGC 6824 (right panel). Bottom: the same for UGC 11919;

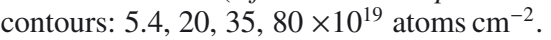

Table 2. H I observational parameters and description of data cubes used in this study (see Sect. 2).

\begin{tabular}{lcc}
\hline \hline & NGC 6824 & UGC 11919 \\
\hline Observing date & 11.08 .2011 & 30.09 .2011 \\
Array configuration & Maxi-short & Maxi-short \\
On-source integration time (h) & 12 & 12 \\
Scale (pix /") & 3.5 & 3.5 \\
Channel width $\mathrm{d} v\left(\mathrm{~km} \mathrm{~s}^{-1}\right)$ & 8.24 & 8.24 \\
Velocity resolution $F W H M_{\mathrm{v}}\left(\mathrm{km} \mathrm{s}^{-1}\right)$ & 16.5 & 16.5 \\
Beam size (") & $19.3 \times 16.5$ & $24.86 \times 16.49$ \\
Beam position angle $\left({ }^{\circ}\right)$ & -0.36 & 0.48 \\
Velocity range $\left(\mathrm{cz}, \mathrm{km} \mathrm{s}^{-1}\right)$ & $2400-4356$ & $4367-6348$ \\
\hline
\end{tabular}

resulting gains were then copied to the original data sets. For the final continuum subtraction, we first subtracted the continuum model resulting from the selfcal procedure directly from the visibilities, to then perform a second-order continuum subtraction, again on the visibilities. The resulting continuum-subtracted visibilities were then inverted to obtain the data cubes. We produced data cubes with different weighting schemes. The cubes that finally adopted were gridded and inverted using a robust weighting of 0.4 and averaged over four channels, representing the most suitable scheme for our aims (to obtain the rotation curve). After the inversion we applied a Hanning smoothing. Finally, the obtained H I data cubes were deconvolved, using the Clean algorithm with clean masks centered on the emission, iteratively decreasing the clean cutoff and increasing the size of the mask regions.

Employing our clean masks, we generated total intensity maps and first-moment maps as shown in Fig. 1, which also shows the optical images with overplotted H I total-intensity maps. From Fig. 1 it is evident that UGC 11919 possesses an
Table 3. Photometric observations: exposure times.

\begin{tabular}{lcc}
\hline \hline & NGC 6824 & UGC 11919 \\
\hline Observing dates & 28.05 .2012 & 27.09 .2011 \\
& 30.05 .2012 & \\
Exposure time (s) $B$ & 5400 & 3000 \\
Exposure time (s) $V$ & 5400 & 1800 \\
Exposure time (s) $R$ & 5400 & \\
\hline
\end{tabular}

extended $\mathrm{H}$ I disk reaching beyond the optical body of the galaxy, while for NGC 6824 the size of the gaseous disk is comparable to that of the optical disk.

\subsection{Photometric observations}

Photometric observations of NGC 6824 and UGC 11919 were made with the 0.5-m Apache Point Observatory telescope using the $B, V$, and $R$ bands. The galaxies were observed under photometric conditions in September 2011 and May 2012. The photometric data reduction was conducted following a standard procedure using the MIDAS software package. The images of photometric standard stars from Landolt $(1992,2009)$ were obtained during the same nights and were used to calibrate the data. A bias and flat field correction was applied to the data. The foreground stars were removed from the galactic images and replaced by the mean fluxes of surrounding regions. The integration times are specified in Table 3.

\section{Results}

We study two galaxies, NGC 6824 and UGC 11919, which, according to preliminary studies, have unusually high (NGC 6824) and low (UGC 11919) M/L ratios for their color indices. We use 

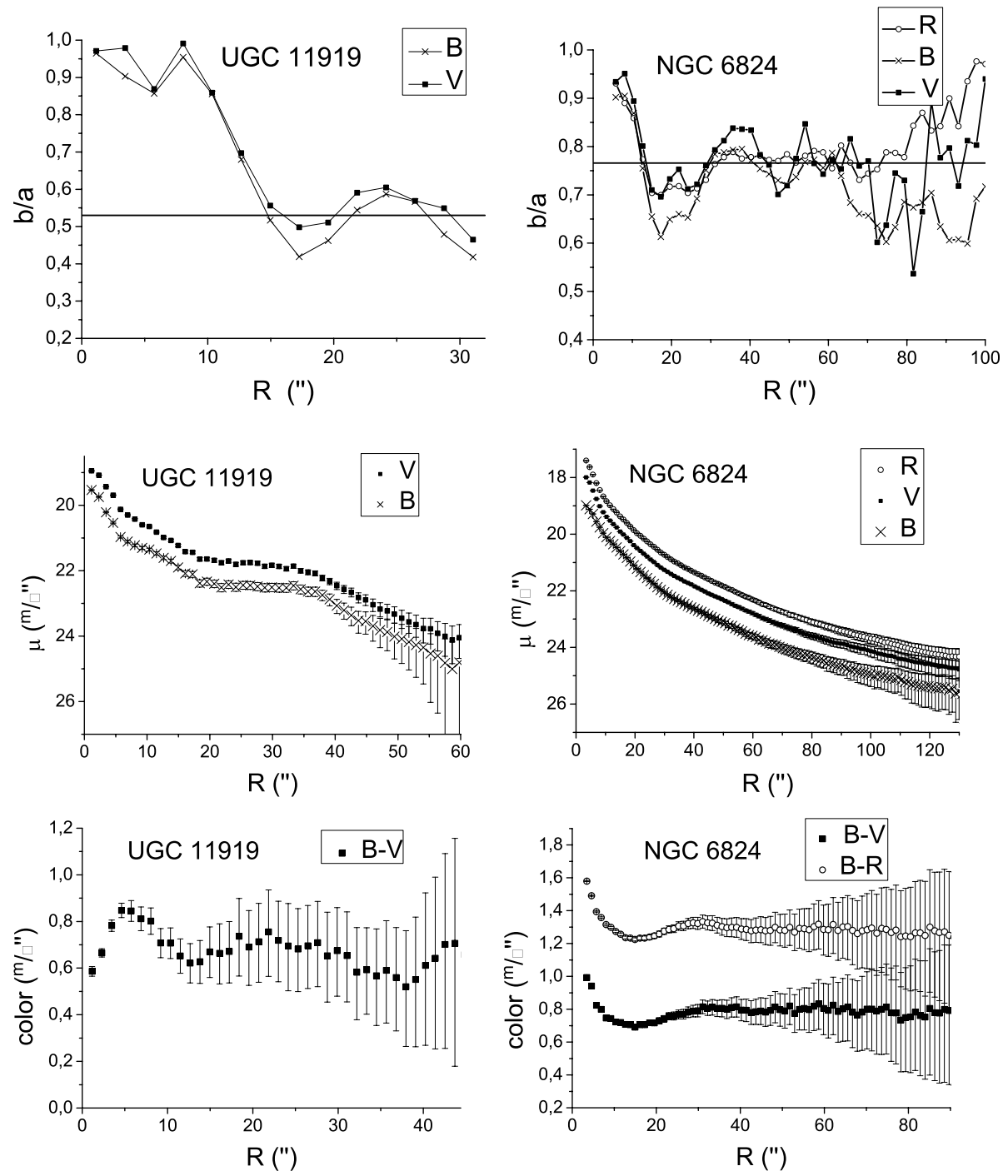

Fig. 2. Radial profiles of isophotal flattening obtained in the $B, V$, and $R$ bands. The horizontal lines correspond to the inclinations $i=58^{\circ}$ and $i=40^{\circ}$ for UGC 11919 and NGC 6824, respectively.
Fig. 3. Azimuthally averaged radial profiles of surface brightness (in $B, V$, and $R$ bands) and color indices. the photometrical data and $\mathrm{H}$ I data cubes to prove or disprove the peculiarity of these objects.

\subsection{Photometric results}

Using the MIDAS software package we obtained the azimuthally averaged profiles of isophotal flattening ( $b / a$, Fig. 2$)$ and radial profiles of surface brightness and color indices for both galaxies (Fig. 3). The profiles are corrected for Galactic extinction according to Schlegel et al. (1998), but are not corrected for internal extinction and disk orientation.

From the isophotal flattening (Fig. 2) we derive an average inclination $i=a \cos (b / a)=40^{\circ} \pm 13^{\circ}$ for NGC 6824 in the radial range $30^{\prime \prime}<R<70^{\prime \prime}$, which is close to the value obtained after masking the spiral arms. For UGC 11919 we derive $i=$ $58^{\circ} \pm 4^{\circ}$ for $R>15^{\prime \prime}$, which changes substantially after masking the prominent spiral arms in the innermost region. In that case we derive $i \geq 35^{\circ}$. The values obtained from isophotal fitting without correcting for the appearance of spiral structure are in good agreement with those given in Hyperleda (see Table 1). Errors for the surface brightness and colors (see Fig. 3) were calculated following Vader \& Chaboyer (1994)

$\delta I=\sqrt{N_{\mathrm{tot}}+\left(\delta n_{\mathrm{sky}} A\right)^{2}}$, where $N_{\text {tot }}$ is the total flux in the aperture of size $A$ and $\delta n_{\text {sky }}$ is the standard deviation of sky background flux obtained in small apertures in the vicinity of a galaxy.

For UGC 11919 the radial profile of the $(B-V)_{0}$ color index is in good agreement with the total value of $(B-V)_{0}$ given in Hyperleda (see Fig. 3 and Table 1). This means that the tentative peculiar value of $\mathrm{M} / \mathrm{L}$ of the galaxy is not a result of incorrect photometrical data given in Hyperleda. For NGC 6824, we find the total color index $(B-V)_{0}=0.74 \pm 0.08$ (corrected for extinction) which differs by 0.09 from the value given in Hyperleda (see Table 1).

To construct dynamical models of the galaxies we require the structural parameters of their bulges and disks. For that purpose we decomposed the images into two components: a disk with an exponential radial brightness distribution $\mu_{\mathrm{d}}(R)=$ $\mu_{0}+1.086 R / R_{\mathrm{d}}$ and a Sersic bulge $\mu_{\mathrm{b}}(R)=\mu_{\mathrm{e}}+c_{\mathrm{n}}\left(\left(R / R_{\mathrm{e}}\right)^{1 / n}-1\right)$. We used BUDDA (de Souza et al. 2004, version 2.2), a dedicated standalone software, to perform this bulge-disk decomposition. The results are shown in Fig. 4. The model and the observed $V$-band images are shown together with the residuals. The images were generated using an identical scale and an identical contrast.

The residual images (Fig. 4) reveal the trace of spiral arms for UGC 11919 and a clear manifestation of an asymmetric structure for NGC 6824. The contrast residuals for NGC 6824 


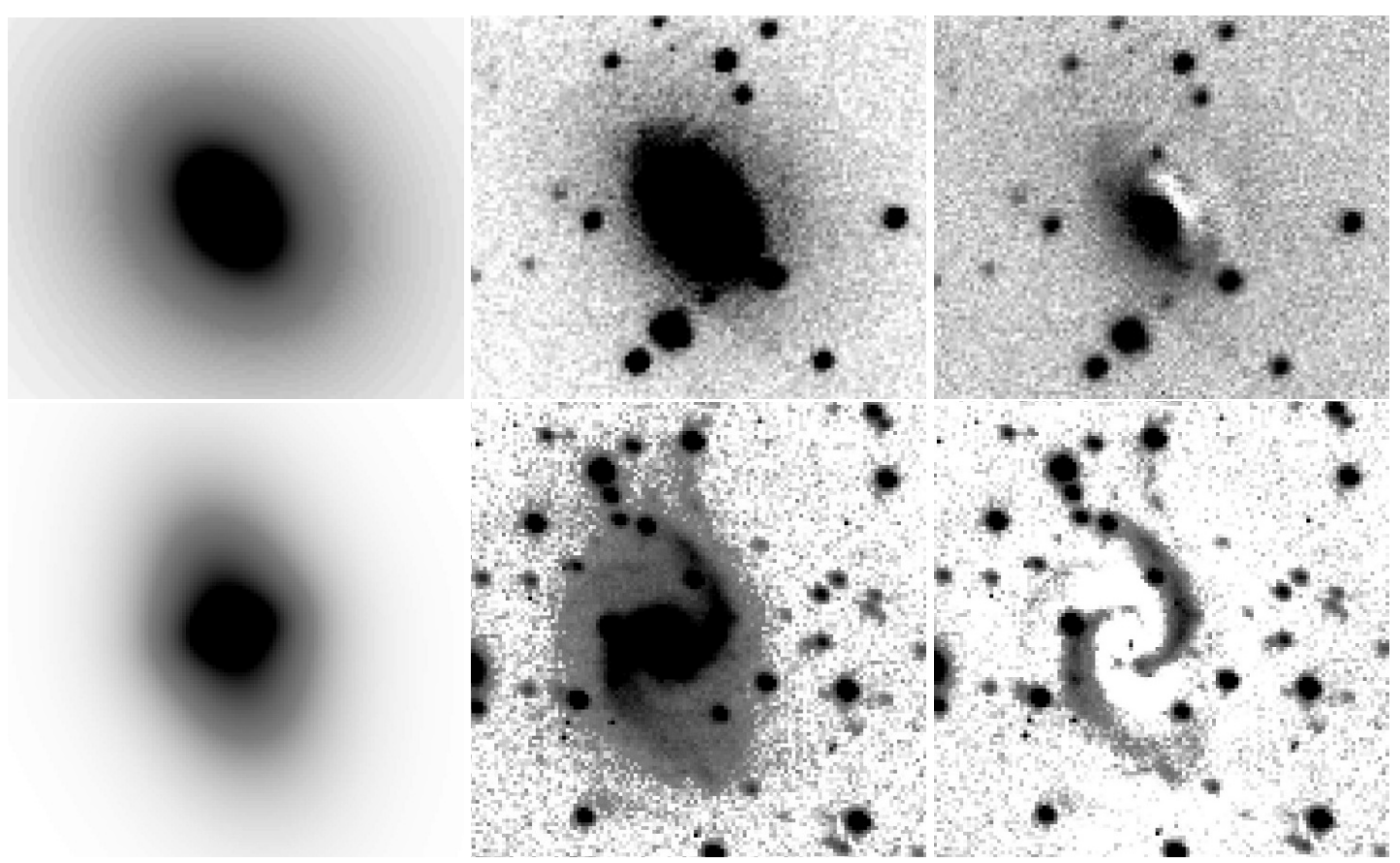

Fig. 4. Results of $V$-band bulge-disk decomposition for NGC 6824 (top) and UGC 11919 (bottom). From left to right: model, observed image, and residual.

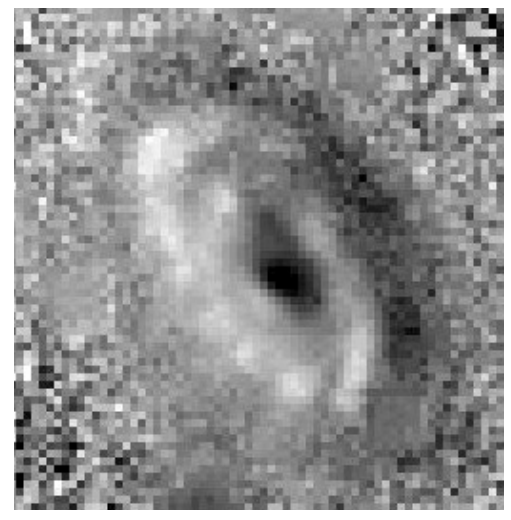

Fig. 5. $B-R$ color map of NGC 6824. The red dust lane (black) is the one most visible above the galaxy center.

are mainly due to a bright spiral arm (dark) together with a dust lane (light) which could not be taken into account in the symmetrical model created by BUDDA. The blue spiral arm is also seen in the $B-R$ color map of the galaxy (see Fig. 5). A red dust lane is also recognizable to the north of the center of NGC 6824 at the top of the galaxy image.

Neglecting the obvious asymmetric features, we derive a deviation of the model from the observed surface brightness profile which does not exceed $0.15 / \square^{\prime \prime}$. With the aid of BUDDA we are able to derive structural models with sufficient quality, despite neglecting local asymmetric features. In Table 4 we list the obtained structural parameters of disk and bulge for the galaxies.

\subsection{Kinematic results}

To compare our photometrical mass estimates with dynamical models (from a mass-decomposition), rotation curves are obtained from our H I data, using tilted-ring modeling. For this purpose, we employ TiRiFiC (Józsa et al. 2007), a software allowing for a direct fit of modified tilted-ring models to data
Table 4. Structural parameters of observed galaxies.

\begin{tabular}{ccccccc}
\hline \hline Galaxy & $\begin{array}{c}R_{\mathrm{d}} \\
\left({ }^{\prime \prime}\right)\end{array}$ & $\begin{array}{c}\mu_{0} \\
\left({ }^{m} / \square^{\prime \prime}\right)\end{array}$ & $\begin{array}{c}R_{\mathrm{e}} \\
\left({ }^{\prime \prime}\right)\end{array}$ & $\begin{array}{c}\mu_{\mathrm{e}} \\
\left({ }^{m} / \square^{\prime \prime}\right)\end{array}$ & $n$ & Filter \\
$(1)$ & $(2)$ & $(3)$ & $(4)$ & $(5)$ & $(6)$ & $(7)$ \\
\hline NGC 6824 & 27.7 & 21.3 & 10.3 & 20.4 & 1.1 & $B$ \\
& 27.1 & 20.6 & 10.5 & 19.8 & 1.1 & $V$ \\
& 25.8 & 19.8 & 8.9 & 18.9 & 1.3 & $R$ \\
\hline UGC 11919 & 20.6 & 21.6 & 9.6 & 22.3 & 2.3 & $B$ \\
& 19.4 & 20.7 & 8.7 & 21.1 & 2.3 & $V$ \\
\hline
\end{tabular}

Notes. (1) Name; (2) exponential disk radial scalelength $R_{\mathrm{d}}$; (3) central surface brightness of the disk; (4) effective radius of the bulge; (5) effective surface brightness of the bulge; (6) Sersic index of the bulge; (7) observing band.

cubes. Figures 6 and 7 show a comparison of data and models for selected images of the H I data cubes. Blue and pink (green) (black and grey (light grey) in printed version) contours represent the $0.75,1.5$, and $3 \mathrm{mJy} /$ beam levels of the observed and model data cubes, respectively. As described below, for UGC 11919 two alternative model data cubes were created. Selected images of the data cubes demonstrating the difference between the two models are shown in Fig. 7.

The data cube of UGC 11919 indicates strong deviations from a flat disk with circular rotation. This is evident from the moment maps, which quite prominently show an inner kinematical twist (Fig. 1, right panel), as well as an outer ring, possibly an extension of the spiral arms of the galaxy (Fig. 1, middle panel). We consider two possibilities for this appearance. In a first attempt to explain this structure, a classical tilted-ring model is fitted where the inclination and the position angle vary with radius, hence representing a symmetric S-shaped warp (see the resulting radial variation of the model parameters in Fig. 8). Using this approach, it is assumed that the apparent deviation from cylindric symmetry, best seen in the total-intensity map and the velocity field, is a projection effect. Comparing model 

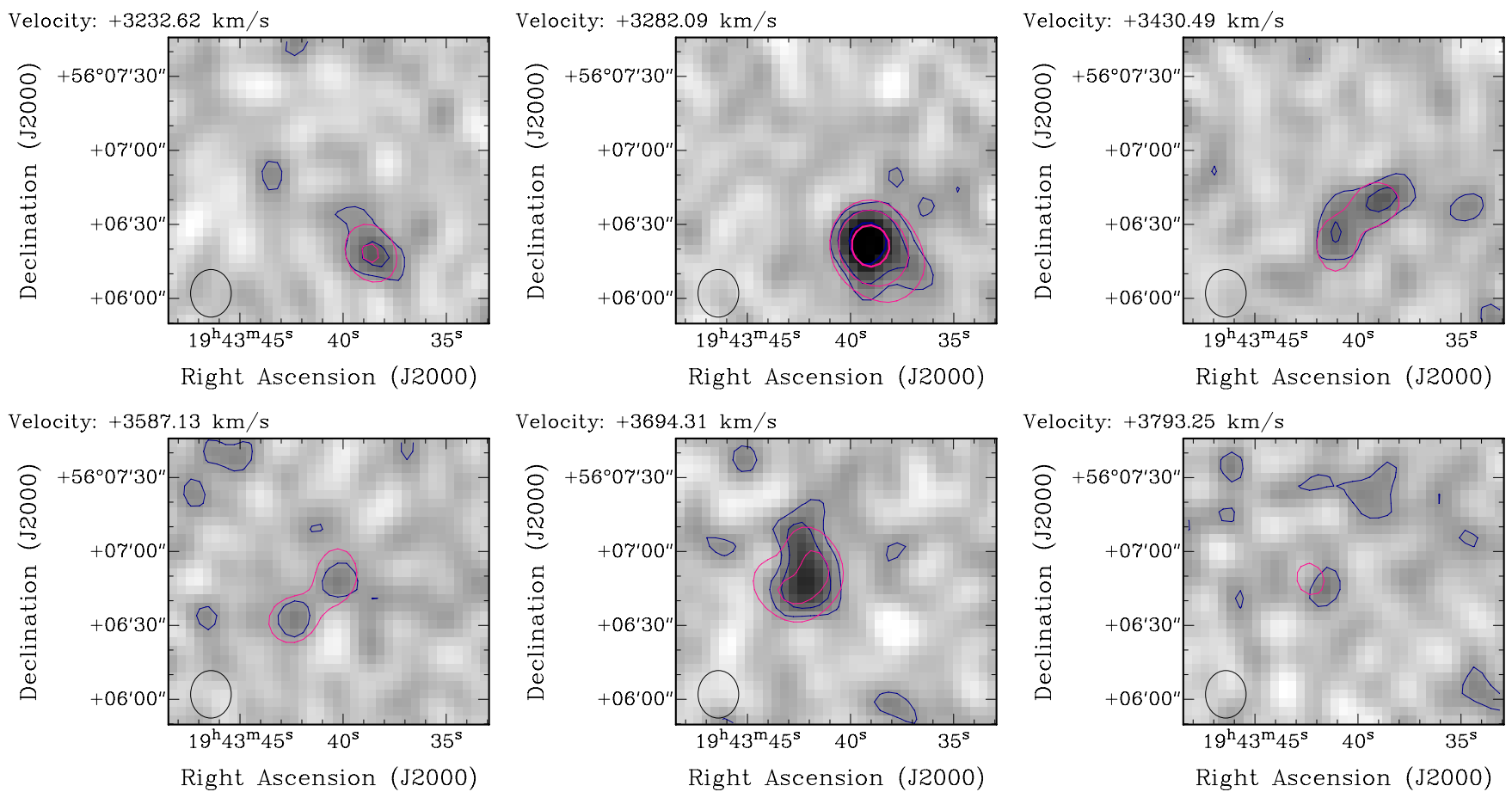

Fig. 6. H I data cubes of NGC 6824. Blue and pink (black and grey in printed version) contours represent the 0.751 .5 , and $3 \mathrm{mJy} / \mathrm{beam}$ levels of the observed and the model data cube.
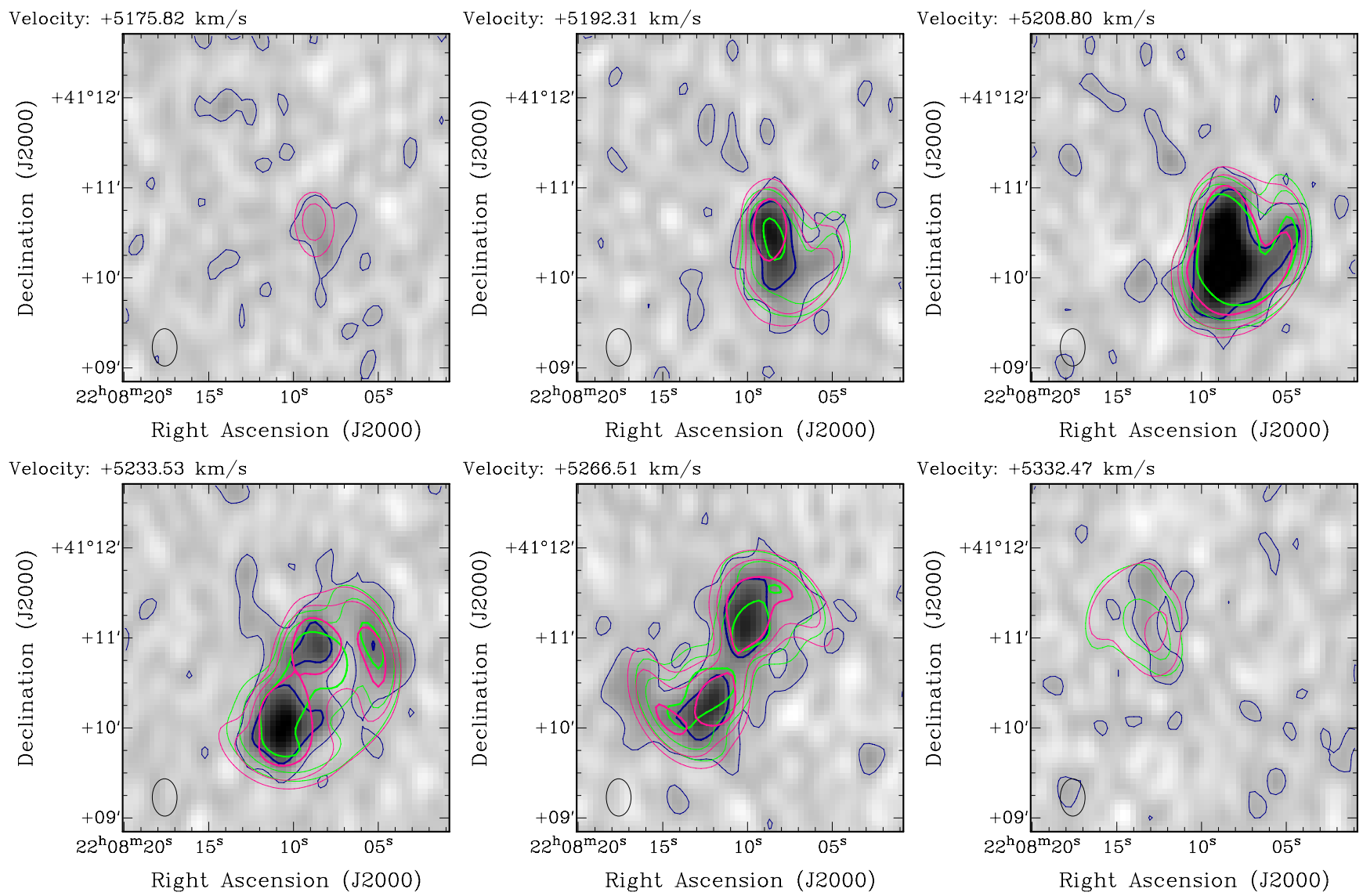

Fig. 7. Selected images of H I data cubes of UGC 11919. Blue, pink, and green (black, grey, and light grey in printed version) contours represent the $0.75,1.5$, and $3 \mathrm{mJy} /$ beam levels of the observed, bisymmetric, and warped model data cubes, respectively.

and observed data cubes, we achieve a good fit. As expected, the model shows a strong warp in the center (reflected by a strong radial change in the inclination and position angle). However, one of the consequences is that the position angle of the H I model deviates strongly from our isophotal fits. The position angle found from isophotal fitting after masking the spiral structure 

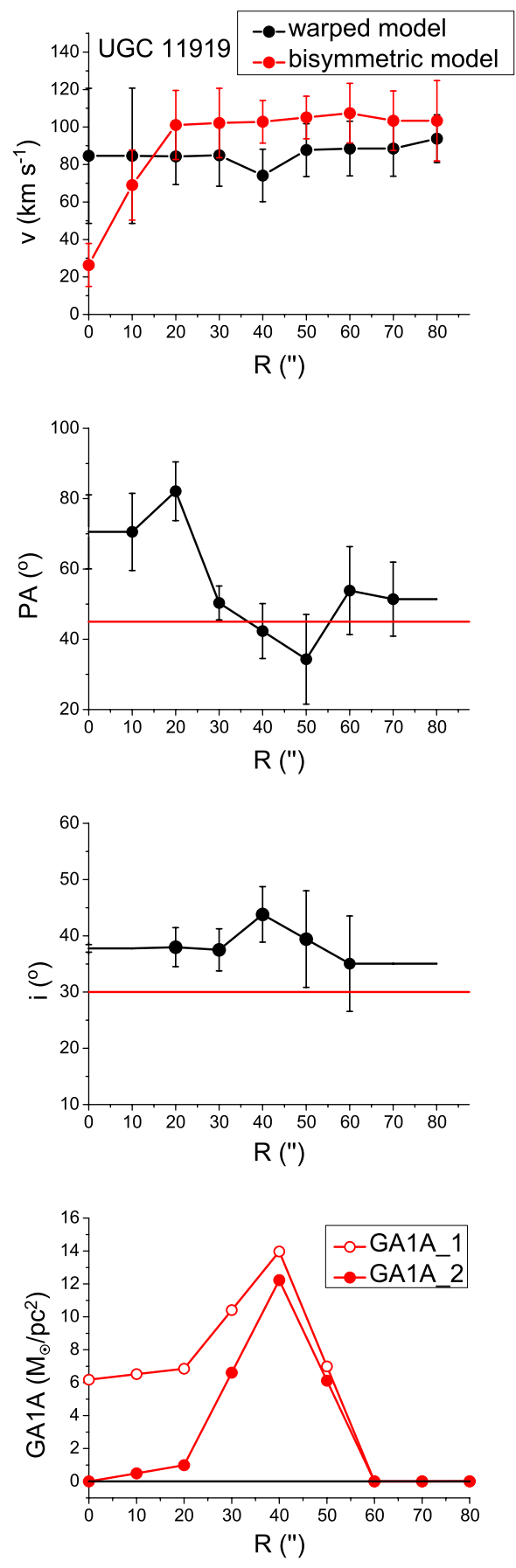
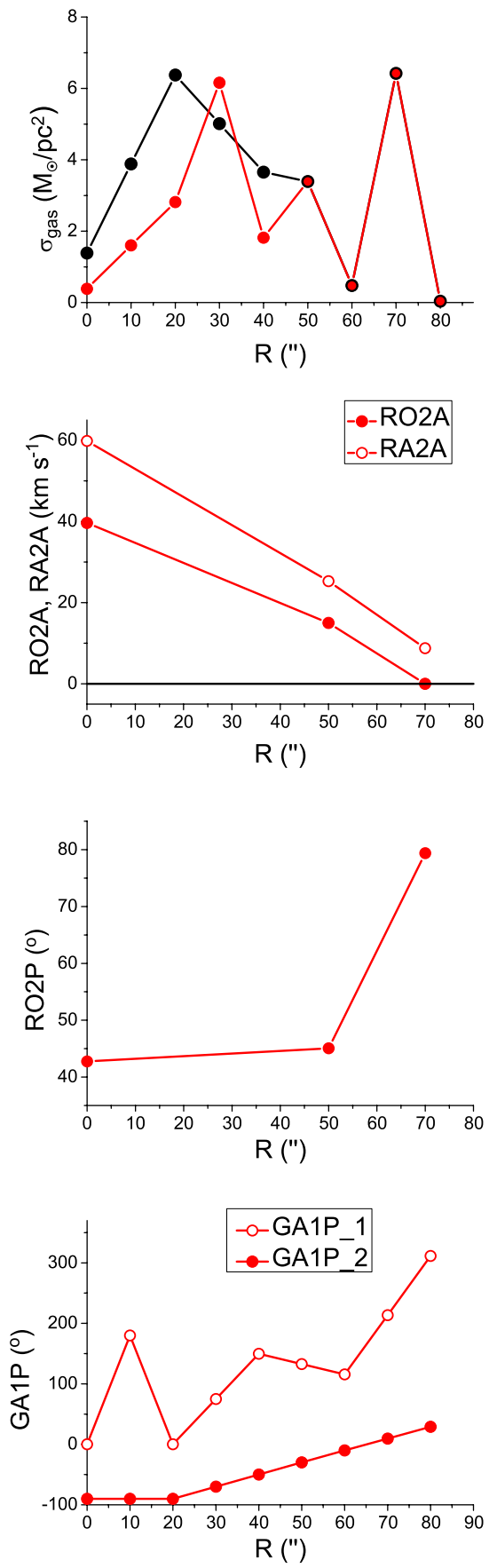

Fig. 8. Radial variation of parameters resulting from the $\mathrm{HI}$ data cube modeling for UGC 11919 for the warped model (black) and bisymmetric model (red in online version, grey in printed version). Surface gas densities and velocity curves are by construction corrected for inclination. RA2A and RO2A are the amplitudes of the second order harmonics of radial and tangential velocity, $\mathrm{RO} 2 \mathrm{P}$ is the phase of RO2A. G1A is the amplitude of a Gaussian surface-brightness distortion representing two spiral arms and G1P is the phase (azimuthal angles) of this distortion. is $\mathrm{PA} \approx 20^{\circ}$, whereas from kinematical modeling we obtain $\mathrm{PA}>40^{\circ}$ (see Fig. 8).

The second modeling approach is to consider that the galaxy possesses a prominent spiral structure and a bar in the center as can be seen from the optical image (see middle panel in Fig. 1). In that picture, one can also see the apparent extension of the optical spiral structure in HI. We tested whether the galaxy can also be modeled as a flat disk in the presence of i) noncircular motions or bar streaming and ii) morphological deviations from cylindric symmetry. Because we have a limited number of data points to compare our model to, we require a simplified approach. For the kinematics we use a model representing the effects of a bisymmetric potential distortion on the kinematics as introduced by Spekkens \& Sellwood (2007) (see also Schoenmakers et al. 1997 and Franx et al. 1994 for more general implementations). In TiRiFiC we reproduce this model by introducing second order harmonic terms in radial and tangential velocity with amplitudes RO2A and RA2A and phases $R A 2 P=R O 2 P+45^{\circ}$, respectively. Other than Spekkens $\&$ Sellwood (2007), we allow the orientation angle of the bisymmetric distortion to vary with the radius. In addition, to introduce azimuthal surface-brightness variations we include a Gaussian surface density distortion with a radially dependent amplitude and a radially dependent phase (see Fig. 8). The dispersion of this distortion was kept constant at $8^{\prime \prime}$. We use this possibility to approximate the H I spiral arms. The phases of the density distortion related to the spiral structure (describing the azimuthal angle of the maximum of the distortion) were found by iteration. They agree well enough with the orientation of the optical spirals (see Fig. 9, left panel). 

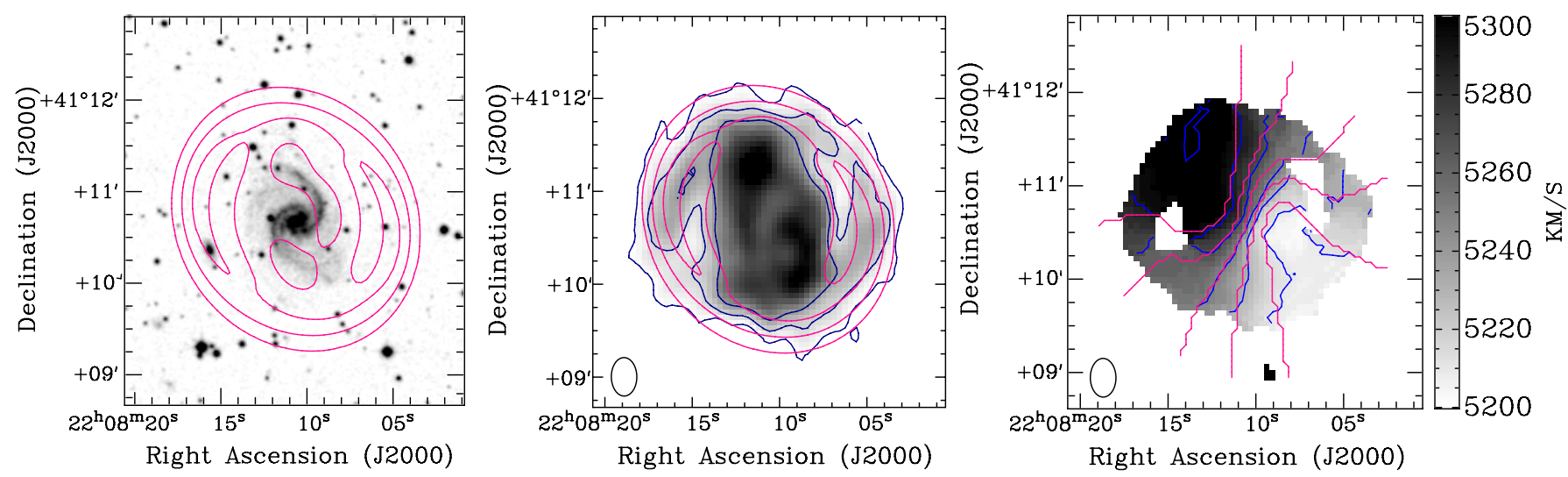

Fig. 9. Left panel: $R$-band optical image of UGC 11919 and H I total intensity with overplotted contours of the bisymmetric model (pink in online version, grey in printed version) H I total intensity: 5.4, 20,35 and $80 \times 10^{19}$ atoms cm${ }^{-2}$. Middle panel: the comparison of observed and bisymmetric model intensity maps with $\mathrm{H}$ I total intensity contours: $5.4,20,35$ and $80 \times 10^{19}$ atoms $\mathrm{cm}^{-2}$. Right: first-moment map with overplotted observed (blue in online edition, black in printed version) and bisymmetric model (pink in online version, grey in printed version) contours $V_{\text {sys }} \pm 0$, 20,40 , and $60 \mathrm{~km} \mathrm{~s}^{-1}$.
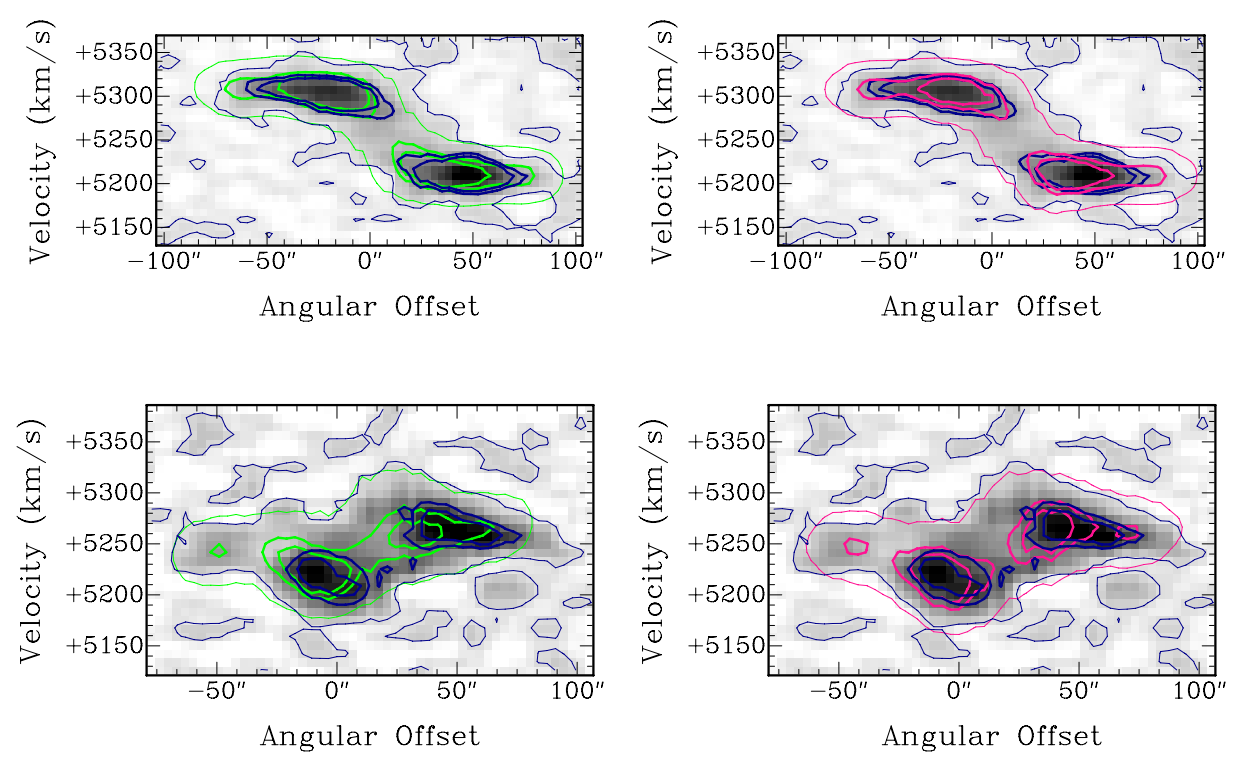

Fig. 10. Position-velocity diagrams along major (top) and minor kinematical axes (bottom) of UGC 11919. The blue, pink, and green (black, grey, and light grey in printed version) lines represent the $0.5,3$, and $4 \mathrm{mJy} / \mathrm{beam}$ levels of the observed and model data cubes, respectively. Left and right panels correspond to the warped model and the model including non-circular motions, respectively.

In Fig. 10 we show the PV-diagrams along the major and minor kinematical axes with overplotted contours of $\mathrm{H}$ I intensity of observed (blue in online edition, black for printed version) and model (pink and green in online version, grey and light grey for printed version) data cubes. The left and right panels correspond to the warped model and the bisymmetric model, respectively. As can be seen from a direct comparison of data cube and models in Fig. 7, a flat disk model provides a better agreement with the observed data cube. The same conclusion may be reached from the comparison of PV-diagrams: a flat disk model gives a better representation of the observed data along the minor axis. Finally, in Fig. 9 we show a comparison of the observed moment maps with the corresponding moment maps for the bisymmetric model to show the good agreement.

We point out that both models provide at least a reasonable fit to the data, such that a final decision which of these very different physical interpretations to adopt is not straightforward. For our analysis we adopt the rotation curve corresponding to the model using the bisymmetric distortion, because of a better correspondence of the data with the model (as concluded by visual inspection), and a more consistent physical picture, since streaming motions are expected in the presence of a bar and strong spiral arms, and also surface density variations following the spiral pattern are expected not to be the result of projection effects only.

A comparison of the warped and bisymmetric model parameters demonstrated in Fig. 8 shows that, as expected, some model parameters are quite different: the central inclination and position angle of the warped disk model are higher than the uniform parameters of the bisymmetric model (inclination $i_{\mathrm{bi}}=$ $30^{\circ} \pm 0.5^{\circ}$, position angle $\mathrm{PA}_{\mathrm{bi}}=45^{\circ} \pm 4^{\circ}$ ). Nevertheless, the resulting rotation curves, the most important derived quantity used in our further analysis, are comparable within the errors (see Fig. 8), such that we conclude that our following analysis is surprisingly not heavily affected by the tilted-ring- model assumptions.

Since in this work we concentrate on the interpretation of the rotation curve only, and fit noncircular motions to disentangle their kinematical signatures rather than using the fitting results to further constrain the dynamics, we refrain from an interpretation of the corresponding coefficients.

Errors were derived by fitting all parameters for the receding and the approaching side independently and calculating their deviation from the best-fit model. Final errors were estimated by averaging the deviations of three neighboring data points. 
The inclination angle of UGC 11919 found from the H I data cube modeling $\left(i=30^{\circ} \pm 0.5^{\circ}\right)$ differs significantly from the angle obtained from the isophotal fitting to the optical image $\left(i=58^{\circ} \pm 4^{\circ}\right)$. The agreement becomes better when the optical spiral structure is masked $\left(i \geq 35^{\circ}\right)$. The position angle from the $\mathrm{H}$ I modeling (bisymmetric model) is found to be PA $=45^{\circ} \pm 4^{\circ}$. The Uppsala General Catalog of Galaxies (Nilson 1973) gives $\mathrm{PA}=35^{\circ}$; our isophotal fitting after masking spiral arms gives an even lower value of $\mathrm{PA} \approx 20^{\circ}$. From the velocity field and the optical image shown in Fig. 1, it can be seen that indeed the kinematical position angle differs from that based on the photometry (even if a judgment based on visual inspection can be misguided by the signatures of noncircular motion). Given that for the optical estimates for the orientation of the galaxy a large fraction of the data has to be masked, we trust the values derived from the $\mathrm{H}$ I to be the more reliable. In the following we use position angle PA and inclination $i$ based on the H I analysis for the quantities derived from the H I data cube (H I surface density profile and rotation curve), but the optically derived inclination and position angle for the stellar surface brightness and color index. We remark that adopting the same (kinematically derived) inclination and position angle for all components does not change our results. The potential resulting decrease in the scalelength of the surface brightness in this case is not strong enough to alter any of our conclusions.

For NGC 6824 we model the H I surface brightness of the receding and the approaching side independently. To restrict the number of free parameters, we assume both position angle and inclination to be constant with radius. The galaxy is close to edge-on, and does not show any obvious variation in position angle by visual inspection of the H I data, such that such an assumption should not affect our derived rotation curve by a significant factor. The rotation velocity and an $m=2$ azimuthal distortion in the line-of-sight velocity (corresponding to the TiRiFiC parameter VM2A, see Fig. 11), which we included to achieve a good fit of the H I cube, were fitted at independent radii for two sides simultaneously. The $m=2$ azimuthal distortion in the line-of-sight velocity is indicative of non-circular motions and basically corresponds to a lopsided rotation curve. With the introduction of a harmonic term, we attempt to disentangle the asymmetric contribution from the rotation curve representing the (cylindrically symmetric part of the) potential.

A satisfactory model corresponds to a constant inclination $i=59^{\circ} \pm 0.5^{\circ}$ and a constant position angle $\mathrm{PA}=45^{\circ} \pm 2.5^{\circ}$, respectively. Errors in inclination, position angles, and rotation velocity were obtained by fitting all these parameters for two sides independently and calculating their deviation from the best-fit model. The resulting rotation curve appears to be in good agreement with that obtained by the GHASP project (Epinat et al. 2008) when corrected for the same inclination angle (see Fig. 12, right).

The inclination found from data cube modeling differs from that obtained from (optical) isophotal flattening for NGC 6824 ( $i=59^{\circ} \pm 0.5^{\circ}$ and $i=40^{\circ} \pm 13^{\circ}$, respectively), which may result from strong antisymmetric optical features in the galaxy. We note that the inclination $i=59^{\circ}$ is in better agreement with the Tully-Fisher relation (see Fig. 14) and the photometric model of the rotation curve. Again, we therefore tend to assign a larger credibility to the inclination derived in our kinematical model.

\subsection{Mass decomposition}

Employing our rotation curves and photometrical data we constructed mass models for UGC 11919 and NGC 6824. For
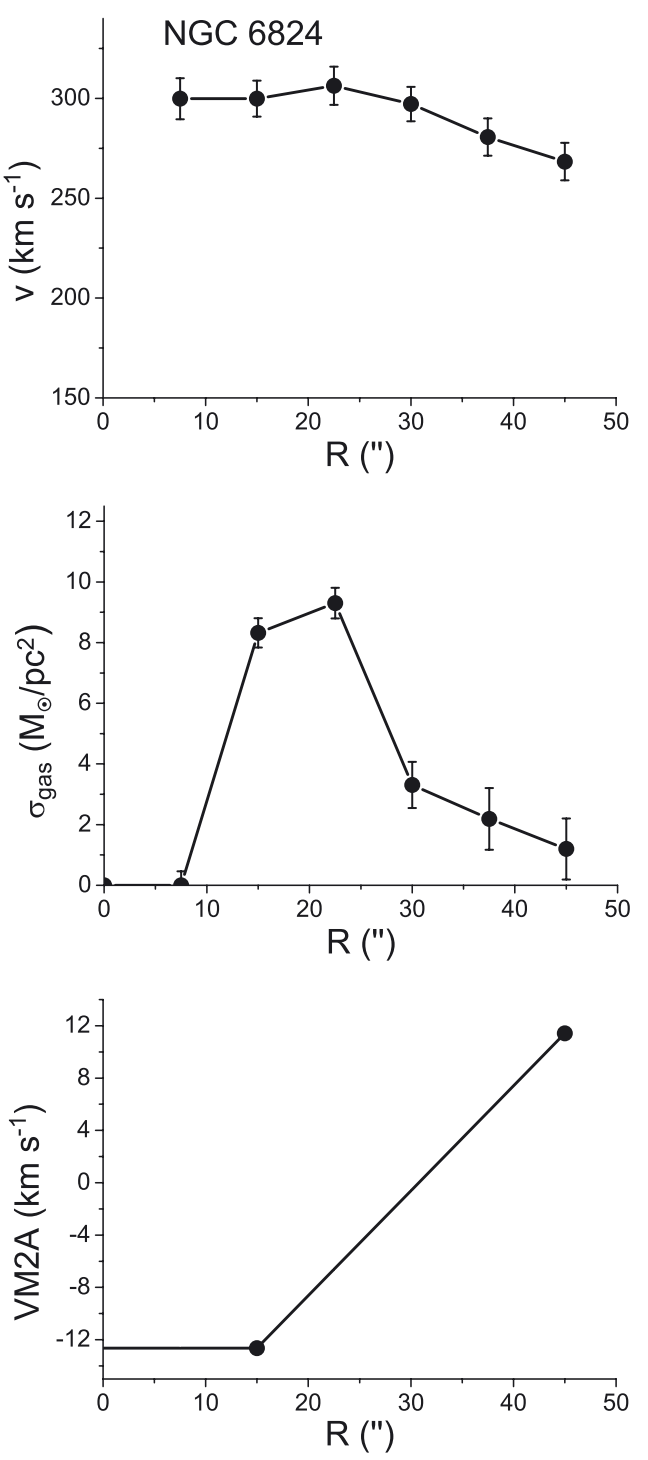

Fig. 11. Radial variation of parameters resulting from the $\mathrm{H}$ I data cube modeling for NGC 6824. VM2A is the amplitude of the adopted second order azimuthal harmonics of the line-of-sight velocity for NGC 6824. Surface gas density and velocity curves are corrected for inclination by construction.

UGC 11919, the stellar M/L of bulge and disk varies with radius, following the variation of their color indices. To fit the data, it is necessary to scale down the surface densities of stellar disk and bulge, because the photometric data together with the standard $\mathrm{M} / \mathrm{L}$-color relation do not agree with the observed rotation curve (see below).

The parameters describing the dark matter halo (radial scale and asymptotic velocity) of UGC 11919 were scaled to achieve the minimal deviation of the resulting model rotation curve from the observed curve. In this paper, we present our results employing a pseudo-isothermal dark halo. The situation does not change when using as an alternative a profile obtained by Navarro et al. 1996 (NFW). Indeed, the difference between the shapes of rotation curves for pseudo-isothermal and NFW halos is significant only in the innermost parts of optical disks (see, e.g., models by de Blok et al. 2008, based on the THINGS H I survey), so the total masses of these two halo types which fit the rotation velocities far from the center, are very close. The accuracy of our derived rotation curve does not allow us to distinguish between 


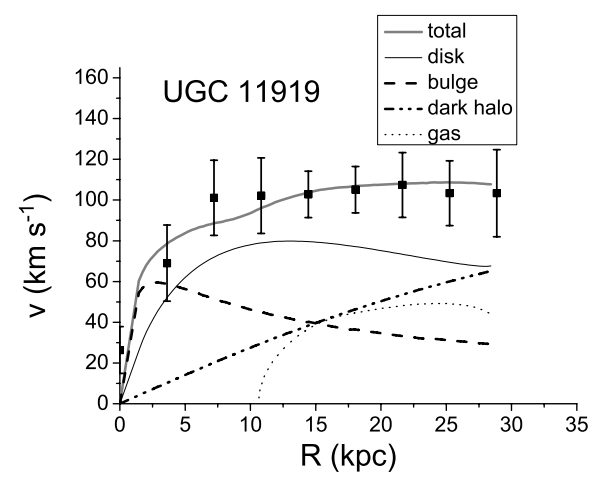

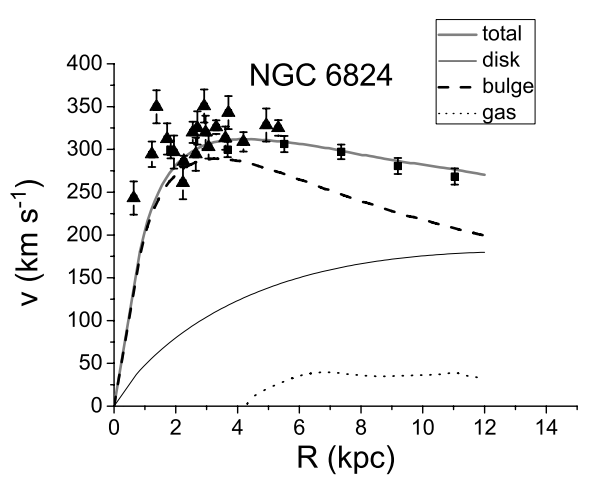

Fig. 12. Left: best-fit model of the H I rotation curve of UGC 11919. Right: model of the combined $\mathrm{HI}+\mathrm{H}_{\alpha}$ rotation curve (squares + triangles) of NGC 6824. The $\mathrm{H}_{\alpha}$ rotation curve is taken from Epinat et al. (2008).

Table 5. Modeling results.

\begin{tabular}{lcccccccc}
\hline \hline $\begin{array}{l}\text { Name } \\
(1)\end{array}$ & $\begin{array}{c}R, \\
(2)\end{array}$ & $\begin{array}{c}M_{\text {disk }} \\
(3)\end{array}$ & $\begin{array}{c}M_{\text {halo }} \\
(4)\end{array}$ & $\begin{array}{c}M_{\text {bulge }} \\
(5)\end{array}$ & $\begin{array}{c}M_{\text {gas }} \\
(6)\end{array}$ & $\begin{array}{c}M_{\text {tot }} \\
(7)\end{array}$ & $\begin{array}{c}\left(M / L_{B}\right)_{\text {disk 1 }} \\
(8)\end{array}$ & $\begin{array}{c}\left(M / L_{B}\right)_{\text {disk 2 }} \\
(9)\end{array}$ \\
\hline NGC 6824 & 14 & 8.0 & 0.0 & 11 & 0.2 & 19 & $2.5 \pm 0.3$ & 2.7 \\
UGC 11919 & 15 & 2.0 & 0.5 & 0.7 & 0.4 & 3.6 & $1.7 \pm 0.3$ & 0.5 \\
UGC 11919 & 29 & 2.4 & 2.9 & 0.7 & 0.9 & 6.9 & $1.7 \pm 0.3$ & 0.5 \\
\hline
\end{tabular}

Notes. (1) Name; (2) radius $R$ for the mass estimate (kpc); (3) disk mass inside $R\left(10^{10} M_{\odot}\right)$; (4) dark matter halo mass inside $R\left(10^{10} M_{\odot}\right)$; (5) bulge mass inside $R\left(10^{10} M_{\odot}\right)$; (6) gaseous disk mass inside $R\left(10^{10} M_{\odot}\right)$; (7) total mass inside $R\left(10^{10} M_{\odot}\right) ;(8)$ disk $\mathrm{M} / \mathrm{L}$ obtained from the observed $(B-V)$ color of the disk, according to Bell \& de Jong (2001); (9) disk M/L corresponding to the best-fit model of the rotation curve.

the cases of pseudo-isothermal and NFW halos. The density profile of the H I disk was taken from the observations and scaled by 1.3 to take into account helium in both models.

For UGC 11919 no additional kinematical information is available from the literature. The best-fit model of our rotation curve is consistent with a mass of the stellar disk that is three times lower (see Table 5) and a bulge mass that is five times less massive than expected from the photometry for the given color (the mean stellar mass-to-light ratio of the bulge is $\left(M / L_{B}\right)_{\text {bulge dyn. }}=0.4$ in our model, while $\left(M / L_{B}\right)_{\text {bulge phot. }}=2$ is deduced from the photometry ). The best-fit model is shown in Fig. 12 (left). The dynamically (1) and photometrically (2) determined mass-to-light ratios of the disk $\left(M / L_{B}\right)_{\text {disk } 1}\left(M / L_{B}\right)_{\text {disk } 2}$ are given in Table 5 .

The maximum rotational velocity of the disk component of the rotation curve of UGC 11919 estimated from the photometric disk mass and the disk scalelength can be calculated via $v_{\text {max disk }} \approx 0.623\left(G M_{\mathrm{d}} / R_{\mathrm{d}}\right)^{0.5}$, where $M_{\mathrm{d}}=2 \pi G \sigma_{0} R_{\mathrm{d}}^{2}$ denotes the total disk mass and $\sigma_{0}$ is the disk central surface density. If we assume $\sigma_{0}$ and $R_{\mathrm{d}}$ from our photometry, the expected value of the rotation velocity $v_{\max }$ disk $=159 \pm 22$ at $R \approx 2 R_{\mathrm{d}}$ is significantly higher than the maximum rotation amplitude $v=102 \pm 18$ (see Fig. 8). Therefore, the result does not depend on the used dark halo density profile, because it would persist even if no dark matter were present in the galaxy. The result is also robust against possible errors in the decomposition of the image into bulge and disk components, because the total (disk + bulge) stellar surface density expected for a normal stellar M/L corresponds to a higher rotation velocity than is observed. The situation also does not change if we neglect the innermost two points of the rotation curve, where the kinematics may be dominated by non-circular motions, because the radius at which the disk component with an exponential surface density profile reaches its maximum rotation velocity $(R \approx 10 \mathrm{kpc})$ is beyond this region. Furthermore, as elaborated above, we show that even under the assumption of a strong warp instead of a bisymmetric distortion the rotation curve stays the same within the errors (see Fig. 8). Hence, a standard photometric approach strongly overestimates the stellar mass, and this galaxy most likely has indeed a light stellar component in both disk and bulge with a non-standard IMF. The total dynamical mass-to-light ratio is $\left(M / L_{B}\right)_{\text {tot }}=1.0$ for UGC 11919 inside the optical radius $R_{25}$ (according to our estimates the apparent $B$-band magnitude within $R_{25}$ ), corrected for Galactic extinction is $m_{B}=13.5$. Hyperleda data (see Table 1) lead to an even lower $M / L_{B}$.

For NGC 6824 we achieve a consistent model by using the photometrically determined mass of the stellar components (bulge and disk) for a standard IMF. The previously suspected large $\mathrm{M} / \mathrm{L}$ ratio for this galaxy was evidently a result of an underestimation of the optically determined inclination and the underestimation of the color index given in Hyperleda.

A decomposition of the rotation curve of NGC 6824 for photometrically estimated masses of stellar components is shown in Fig. 12 (right). The presented model is close to the best-fit model, which corresponds to the minimum discrepancy between the observed and model rotation curves. The squares correspond to the H I rotation curve found in this work and the triangles show the $\mathrm{H}_{\alpha}$ rotation curve obtained in the GHASP project by Epinat et al. (2008). From the model it follows that the mass distribution of NGC 6824 is dominated by the visible matter inside a radius of $R=12 \mathrm{kpc}$. Within a radius of $R \approx 2 R_{\mathrm{d}}$, the rotation curve of this galaxy can be explained solely by baryonic matter without the contribution of a DM halo (see Fig. 12).

The total dynamical $\mathrm{M} / \mathrm{L}$ for this galaxy as derived from the mass-decomposition is $\left(M / L_{B}\right)_{\text {tot }}=3.4$ within the optical radius $R_{25}$. This is based on our measurements in which we determined the apparent $B$-band magnitude within $R_{25}$ (corrected for Galactic extinction) to be $m_{B}=12.1$. The model rotation curve of NGC 6824, shown in Fig. 12, corresponds to the stellar mass-to-light ratios of the bulge $\left(M / L_{R}\right)_{\text {bulge dyn. }}=2.5$ and $\left(M / L_{B}\right)_{\text {bulge dyn. }}=3.3$, in good agreement with the values obtained from the color indices $(B-R)_{0}$ and $(B-V)_{0}$ of the bulge and a standard $\mathrm{M} / \mathrm{L}$ - color model relation $\left(\left(M / L_{R}\right)_{\text {bulge phot. }}=\right.$ 2.6 and $\left(M / L_{B}\right)_{\text {bulge phot. }}=3.6$, respectively. The same conclusion is valid for the stellar disk (see Table 5). 


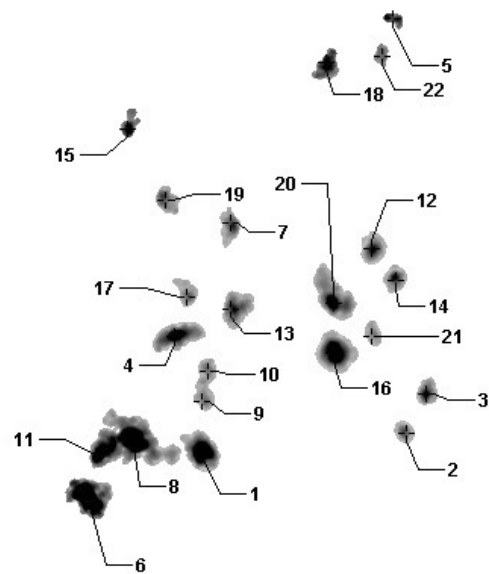

$\mathrm{E}^{\mathrm{N}} \stackrel{15^{\prime}}{1.078^{\circ} \times 1.061^{\circ}}$
Fig. 13. HI intensity maps of the environment of UGC 11919 (left) and NGC 6824 (right), obtained from the data cube with robust weighting of 0.4 , tapering of $30^{\prime \prime}$, and averaged by four channels with the labeled objects.
There are two main sources of systematic errors for dynamically determined masses. These are non-circular gas motion and disk asymmetries, which in turn lead to an uncertainty in the measurement of the inclination, and with that of the rotation velocity and surface-brightness profiles. However, we explicitly include kinematical asymmetries in our models, such that we can assume to minimize errors of this kind. Another source of uncertainties could be related to the correction for the internal extinction. However, the extinction affects the $M / L_{B}-$ color index diagram only slightly, because it increases $M / L$ and the color index simultaneously, and these shifts nearly compensate each other (see, e.g., Bell \& de Jong 2001).

We note that the evolutionary models recently created by Into \& Portinari (2013) where the Thermally Pulsing AGB phase is taken into account, provide optical $\mathrm{M} / \mathrm{L}$ - color relationships which are nearly identical to those obtained earlier by Bell \& de Jong (2001).

Table 5 presents the results of the mass decomposition within the optical radius $R_{25}$ for the galaxies. For UGC 11919 we also give the masses of the components up to the last measured point of the rotation curve.

\section{Environment}

For the targeted galaxies we construct H I total-intensity maps of the environment using the data cubes with a robust weighting of 0.4, a tapering of 30 arcsec, and averaging four channels.

From Fig. 13 (left) a rather dense environment within the bounds of our data cube becomes evident. We derive the main integral parameters, position, systemic velocity, total H I flux, H I mass, and HI mass-to-light ratio ( $B$-band where available) of all objects detected in this cube, shown in Table 6. Because our data cube is limited to a minimum velocity of $4367 \mathrm{~km} \mathrm{~s}^{-1}$, we do not cover the complete velocity range of this group. In Table 6 we mark galaxies, for which the H I lies partly outside the (velocity) bounds of our data cube with an asterisk. Velocities of these objects denote only the averaged velocity of the detected $\mathrm{HI}$ and hence do not reflect the systemic velocity.

Table 6 and Fig. 13 (left) reveal a rich group of galaxies around object 8 (NGC 7223, see Crook et al. 2007) to the south-east of UGC 11919. A simple comparison of velocities shows that UGC 11919 probably does not belong to this group. From Table 6 it is evident that the systemic velocities of detected objects in the data cube cluster in two ranges (objects $1-15$ and objects 16-22). The average velocity (cz) in range 1 (objects $1-15$ ) is $4558 \pm 112 \mathrm{~km} \mathrm{~s}^{-1}$, while in range 2 (objects 16-22) it is $5550 \pm 130 \mathrm{~km} \mathrm{~s}^{-1}$. The systemic velocity of UGC 11919 differs by $800 \mathrm{~km} \mathrm{~s}^{-1}$ from the average velocity in range 1 , which is seven times larger than the standard deviation of the measured velocities in range 1 (which may be used as a proxy for the velocity dispersion in the group, despite the fact that we are not measuring the velocities of each group member correctly, see below) and two times larger than the linking velocity of $350 \mathrm{~km} \mathrm{~s}^{-1}$ that acts as one selection criterion to identify two galaxies as a coupled pair in Crook et al. 2007, (see also Ramella et al. 1997). Selecting by this typical linking velocity (Crook et al. 2007, Ramella et al. 1997) alone, none of the galaxies 1-15 would be identified as belonging to the same group as UGC 11919. We point out that our table does not give a full account of all objects in the group, since it is at the edges of our velocity bounds. However, this result will probably still hold if we were able to take the systemic velocities of objects $1-5$ properly into account, since they are currently biased towards velocities that are too high (closer to UGC 11919).

The galaxy UGC 11919 possesses two companions, object 20 (CGCG 530-011) and object 21 at the projected distances of $r<100 \mathrm{kpc}$. The velocity differences from UGC 11919 are $355 \mathrm{~km} \mathrm{~s}^{-1}$ (20) and $252 \mathrm{~km} \mathrm{~s}^{-1}$ (21). The galaxy CGCG 530-011 has a peculiar H I structure which might originate from an interaction. While our target galaxy is not a member of a rich group, it is also not necessarily an isolated galaxy. The H I ring found for UGC 11919 could hence also be a result of gravitational interaction.

The galaxy NGC 6824 is a member of a group with mean velocity $V_{\mathrm{lg}}=4065 \mathrm{~km} \mathrm{~s}^{-1}$. The asymmetric structure and the $m=2$ distortion in the line-of-sight velocity of this galaxy could be due to the gravitational interaction with the members of the group (see Fig.13, right, and Table 6).

We therefore conclude that neither UGC 11919 nor NGC 6824 is a particularly isolated galaxy. Both show irregular velocity structure, possibly as a result of a recent interaction. Whether this interaction might have led to the peculiar $\mathrm{M} / \mathrm{L}$ for UGC 11919 is questionable. The interaction with neighbor galaxies has already experienced a significant part of all existing galaxies possessing the standard IMF, so by itself it cannot explain the paucity of low-mass stars. It is worth mentioning that 
Table 6. Environmental characteristics of UGC 11919 and NGC 6824.

\begin{tabular}{|c|c|c|c|c|c|c|c|c|}
\hline $\begin{array}{c}\# \\
(1)\end{array}$ & $\begin{array}{l}\text { Object designation } \\
\text { (2) }\end{array}$ & $\begin{array}{l}\text { RA } \\
(3)\end{array}$ & $\begin{array}{l}\text { Dec } \\
\text { (4) }\end{array}$ & $\begin{array}{l}c z \\
(5)\end{array}$ & $\begin{array}{c}\text { H I flux }\left(\mathrm{Jy} \mathrm{km} \mathrm{s}^{-1}\right) \\
\text { (6) }\end{array}$ & $\begin{array}{l}V_{\lg } \\
(7)\end{array}$ & $\begin{array}{c}M_{\mathrm{HI}}\left(10^{9} M_{\odot}\right) \\
(8)\end{array}$ & $\begin{array}{l}M_{\mathrm{HI}_{\mathrm{I}}} / L_{\mathrm{B}} \\
\quad(9)\end{array}$ \\
\hline \multicolumn{9}{|c|}{ UGC 11919} \\
\hline $1^{*}$ & UGC 11927 & $22: 09: 27.86$ & $+40: 59: 56.7$ & 4501 & 7.46 & 4800 & 7.21 & 0.43 \\
\hline $2^{*}$ & & 22:07:30.80 & $+41: 01: 55.4$ & 4398 & 0.44 & 4698 & 0.4 & - \\
\hline $3^{*}$ & & 22:07:17.93 & $+41: 06: 00.1$ & 4448 & 1.16 & 4747 & 1.09 & - \\
\hline $4^{*}$ & \multirow[t]{2}{*}{ 2MASX J22094543+4112200 } & 22:09:43.18 & $+41: 12: 38.5$ & 4536 & 4.78 & 4836 & 4.69 & - \\
\hline $5^{*}$ & & $22: 07: 36.15$ & $+41: 47: 26.8$ & 4388 & 1.15 & 4688 & 1.06 & - \\
\hline 6 & CGCG 530-014 & $22: 10: 34.14$ & $+40: 54: 37.7$ & 4525 & 18.03 & 4824 & 17.6 & 0.81 \\
\hline 7 & UGC 11925 & 22:09:10.35 & $+41: 24: 41.1$ & 4548 & 0.97 & 4848 & 0.96 & 0.16 \\
\hline 8 & NGC 7223 & $22: 10: 08.31$ & $+41: 01: 12.0$ & 4697 & 18.4 & 4997 & 19.3 & 0.3 \\
\hline 9 & & 22:09:26.87 & $+41: 05: 31.7$ & 4465 & 0.36 & 4765 & 0.34 & - \\
\hline 10 & & 22:09:25.16 & $+41: 08: 51.8$ & 4568 & 0.4 & 4868 & 0.4 & - \\
\hline 11 & & $22: 10: 26.16$ & $+40: 59: 44.6$ & 4647 & 7.62 & 4947 & 7.83 & - \\
\hline 12 & & $22: 07: 48.81$ & $+41: 22: 01.3$ & 4620 & 1.43 & 4920 & 1.45 & - \\
\hline 13 & \multirow[t]{3}{*}{ 2MASX J22090972+4115259 } & 22:09:09.99 & $+41: 15: 26.7$ & 4772 & 1.97 & 5072 & 2.12 & - \\
\hline 14 & & 22:07:35.56 & $+41: 18: 57.7$ & 4693 & 1.04 & 4993 & 1.09 & - \\
\hline 15 & & $22: 10: 12.18$ & $+41: 35: 17.2$ & 4703 & 1.77 & 5003 & 1.86 & - \\
\hline 16 & UGC 11919 & 22:08:10.51 & $+41: 10: 47.4$ & 5354 & 7.17 & 5654 & 9.61 & 0.3 \\
\hline 17 & \multirow[t]{3}{*}{ LEDA 3087958} & 22:09:36.12 & $+41: 16: 52.6$ & 5447 & 0.45 & 5747 & 0.63 & 0.07 \\
\hline 18 & & 22:08:15.09 & $+41: 42: 28.9$ & 5499 & 3.23 & 5799 & 4.56 & - \\
\hline 19 & & 22:09:51.82 & $+41: 27: 48.0$ & 5499 & 0.88 & 5799 & 1.24 & - \\
\hline 20 & \multirow[t]{3}{*}{ CGCG 530-011 } & 22:08:12.18 & $+41: 16: 19.6$ & 5709 & 4.63 & 6009 & 7.01 & 0.35 \\
\hline 21 & & 22:07:48.03 & $+41: 12: 45.0$ & 5606 & 0.23 & 5906 & 0.34 & - \\
\hline \multirow[t]{2}{*}{22} & & 22:07:43.38 & $+41: 43: 25.6$ & 5735 & 0.51 & 6035 & 0.78 & - \\
\hline & \multicolumn{8}{|c|}{ NGC 6824} \\
\hline 1 & NGC 6824 & $19: 43: 41.27$ & $+56: 06: 34.0$ & 3563 & 1.88 & 3835 & 1.16 & 0.02 \\
\hline 2 & & 19:44:04.82 & $+56: 29: 03.9$ & 3493 & 0.74 & 3766 & 0.44 & - \\
\hline 3 & LEDA 214725 & $19: 43: 57.39$ & $+56: 04: 33.9$ & 3949 & 0.16 & 4221 & 0.12 & 0.16 \\
\hline 4 & & 19:43:29.69 & $+55: 50: 22.7$ & 4164 & 0.18 & 4436 & 0.15 & - \\
\hline
\end{tabular}

Notes. (1) Number of detected object, as labeled in Fig. 13; (2) Name of object if known; (3) Right ascension J2000; (4) Declination J2000; (5) Optical recession velocity; (6) Total H I flux $F$ in Jy km s${ }^{-1}$; (7) Velocity corrected to the centroid of the Local Group using the formula following Yahil et al. (1977); (8) H I total mass calculated from the total flux via $M=2.36 \times 10^{5}\left(V_{\mathrm{lg}} /\left(75 \mathrm{~km} \mathrm{~s}^{-1}\right)\right)^{2} F$; (9) $\mathrm{H}$ I mass-to-light ratio, the $B$-band magnitudes were taken from Hyperleda. Galaxies with the velocities lying at the edge of the observed data cube (so the systemic velocity is uncertain for them) are marked by asterisks.

the bulk of galactic stars have formed in the past, and so the cause of the existence of abnormally light-weighted disks lays in the properties of cold star-forming gas which existed in the epoch of disk formation, and which are poorly understood now.

\section{Conclusions}

We carried out H I observations together with multicolor surface photometry for two galaxies, UGC 11919 and NGC 6824, for which in a catalog search we found earlier indications of a peculiar $\mathrm{M} / \mathrm{L}$ for a given color index $(B-V)_{0}$. We model the H I data cubes in the scope of an extended tilted-ring model. Using this model we constructed the rotation curves of the galaxies with the aim of deriving their kinematically-based mass distributions.

Both galaxies have some disk asymmetries and rather complex gas kinematics. The main problem in estimating the rotational velocity of NGC 6824 is the ambiguity of its disk inclination angle $i$. An optical isophotal fit gives $i=40^{\circ} \pm 13^{\circ}$, whereas the kinematical fit to the radio data favors $i=59^{\circ} \pm 0.5^{\circ}$. We prefer the kinematical fit, because this galaxy is far from being optically axisymmetric having two open spiral arms and a dust lane. However, if one assumes the lower value for inclination, the rotation velocity would become, under standard assumptions, too high for its luminosity, which in turn could give evidence of a massive dark matter component in the galaxy, provided that the $\mathrm{M} / \mathrm{L}$ ratios of the stellar components correspond to their observed color. However, our trusted value for the inclination, $i=59^{\circ}$, agrees with the $\mathrm{M} / \mathrm{L}$ expected from the photometry.
And there is no firm evidence of any peculiarity of the stellar IMF in this galaxy.

Evidently NGC 6824 is dynamically dominated by visible matter. It possesses an extended pseudobulge and quite normal mass-to-light ratios of disk and bulge. The photometrically determined contributions of stellar disk and bulge are in good agreement with the observed rotation curve. To get a good fit of the H I data cube for NGC 6824 we include an $m=2$ distortion in the line-of-sight velocity.

For the second galaxy, UGC 11919, the situation is different. The analysis of our photometric and kinematic data indicates that the disk of this galaxy, under standard assumptions, is unusually light for its luminosity, independent of the mass of the dark matter halo. Determining the mass of its disk using its $\mathrm{H}$ I kinematics, corrected for non-circular motions, yields a disk mass-to-light ratio of $\left(M / L_{B}\right)_{\text {disk }}=0.5$ in the best-fit model. In this model the rotation curve corresponds to a disk which is three times lighter, and a bulge lighter by a factor of five than is expected from the photometric data. This galaxy may therefore possess a bottom-light stellar mass function. If we assume an inclination found from isophotal flattening $\left(i=58^{\circ}\right)$ instead of the kinematically determined one of $i=30^{\circ}$, we obtain an even lower $\mathrm{M} / \mathrm{L}$ for the galaxy and its components. The employment of an alternative kinematical model reproducing the observations by means of a regularly rotating but heavily warped H I disk also results in a similar rotation curve (with a slightly decreased rotation velocity) and does not, therefore, alter our main conclusion. We also found that this galaxy has an outer $\mathrm{H}$ I ring. It is possible 
A. S. Saburova et al.: Observations and surface photometry of two unusual spiral galaxies

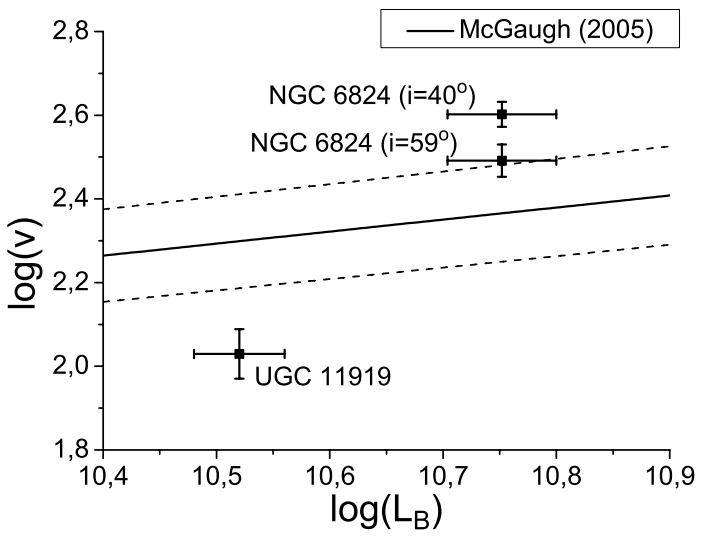

Fig. 14. Position of NGC 6824 (for two values of inclination) and UGC 11919 on the Tully-Fisher diagram (McGaugh 2005). The dashed lines correspond to the formal uncertainties.

that this structure originated from the gravitational interaction with two companions.

Since we determine a luminosity and rotation velocity of our target systems, we are also able to highlight our findings in the context of the classical Tully-Fisher relation (TFR). The term was introduced by Tully \& Fisher (1977) and follow-ups. The TFR appears to hold for a large variety of galactic systems. Any galaxy with an unusual IMF should also be an outlier on this relation. Figure 14 shows that while NGC 6824, for which we found the photometric and dynamically determined masses to be consistent, lies on the upper edges of the relation, UGC 11919 is a clear outlier.

In accordance with our earlier conclusions (Saburova et al. 2011), we claim that spiral galaxies of high brightness with a strongly bottom heavy or bottom light stellar IMF should occur rarely. In the present paper we confirm the existence of a galaxy which has a very low $\mathrm{M} / \mathrm{L}$ for its old (red) stellar population apparently being depleted of low mass stars. These systems deserve a more detailed investigation.

Acknowledgements. We thank the referee, Albert Bosma for his important remarks, which allowed us to improve the paper. We thank Alexei Moiseev for his useful comments. We acknowledge the possibility of using the HyperLeda database (http://www.leda.univ-lyon1.fr/). The photometric data is based on observations obtained with the Apache Point Observatory 0.5-m telescope, which is owned and operated by the Astrophysical Research Consortium. Anna Saburova thanks ASTRON (Netherlands) for the possibility of visiting it and for help with the H I data reduction. The Westerbork Synthesis Radio Telescope is operated by the ASTRON (Netherlands Foundation for Research in Astronomy) with support from the Netherlands Foundation for Scientific Research (NWO). This work was partly supported by Russian Foundation for Basic Research, grants 11-02-12247 and 12-02-31452, and the Dynasty fund. TiRiFiC is publicly available from http://www.astron.nl/ jozsa/tirific/.

\section{References}

Bastian, N., Covey, K. R., \& Meyer, M. R. 2010, ARA\&A, 48, 339 Bell, E. F., \& de Jong, R. S. 2001, ApJ, 550, 212

Bruzual, G., \& Charlot, S. 2003, MNRAS, 344, 1000

Crook, A. C., Huchra, J. P., Martimbeau, N., et al. 2007, ApJ, 655, 790

Dabringhausen, J., Fellhauer, M., \& Kroupa, P. 2010, MNRAS, 403, 1054

de Blok, W. J. G., Walter, F., Brinks, E., et al. 2008, AJ, 136, 2648 de Souza, R. E., Gadotti, D. A., \& dos Anjos, S. 2004, ApJS, 153, 411 Epinat, B., Amram, P., Marcelin, M., et al. 2008, MNRAS, 388, 500 Franx, M., van Gorkom, J. H., \& de Zeeuw, T. 1994, ApJ, 436, 642 Fuchs, B. 2003, Ap\&SS, 284, 719

Gilmore, G. 2001, in Starburst Galaxies: Near and Far, eds. L. Tacconi, \& D. Lutz, 34

Goudfrooij, P., \& Kruijssen, J. M. D. 2013, ApJ, 762, 107

Gunawardhana, M. L. P., Hopkins, A. M., Sharp, R. G., et al. 2011, MNRAS, 415, 1647

Hoversten, E. A., \& Glazebrook, K. 2008, ApJ, 675, 163

Into, T., \& Portinari, L. 2013, MNRAS, 430, 2715

Józsa, G. I. G., Kenn, F., Klein, U., \& Oosterloo, T. A. 2007, A\&A, 468, 731

Kroupa, P. 2002, Science, 295, 82

Kroupa, P. 2012 [arXiv: 1210.1211]

Landolt, A. U. 1992, AJ, 104, 340

Landolt, A. U. 2009, AJ, 137, 4186

Lee, H.-c., Gibson, B. K., Flynn, C., Kawata, D., \& Beasley, M. A. 2004, MNRAS, 353, 113

Lee, J. C., Gil de Paz, A., Tremonti, C., et al. 2009, ApJ, 706, 599

McGaugh, S. S. 2005, ApJ, 632, 859

Meurer, G. R., Wong, O. I., Kim, J. H., et al. 2009, ApJ, 695, 765

Navarro, J. F., Frenk, C. S., \& White, S. D. M. 1996, ApJ, 462, 563

Nilson, P. 1973, Nova Acta Regiae Soc. Sci. Upsaliensis Ser. V, 0

Portinari, L., Sommer-Larsen, J., \& Tantalo, R. 2004, MNRAS, 347, 691

Ramella, M., Pisani, A., \& Geller, M. J. 1997, AJ, 113, 483

Read, J. I., Mayer, L., Brooks, A. M., Governato, F., \& Lake, G. 2009, MNRAS, 397,44

Saburova, A. S. 2011, Astron. Rep., 55, 409

Saburova, A. S., Shaldenkova, E. S., \& Zasov, A. V. 2009, Astron. Rep., 53, 801

Saburova, A. S., Bizyaev, D. V., \& Zasov, A. V. 2011, Astron. Lett., 37, 751

Sault, R. J., Teuben, P. J., \& Wright, M. C. H. 1995, in Astronomical Data Analysis Software and Systems IV, eds. R. A. Shaw, H. E. Payne, \& J. J. E. Hayes, ASP Conf. Ser., 77, 433

Schlegel, D. J., Finkbeiner, D. P., \& Davis, M. 1998, ApJ, 500, 525

Schoenmakers, R. H. M., Franx, M., \& de Zeeuw, P. T. 1997, MNRAS, 292, 349

Spekkens, K., \& Sellwood, J. A. 2007, ApJ, 664, 204

Tully, R. B., \& Fisher, J. R. 1977, A\&A, 54, 661

Vader, J. P., \& Chaboyer, B. 1994, AJ, 108, 1209

Yahil, A., Tammann, G. A., \& Sandage, A. 1977, ApJ, 217, 903

Zackrisson, E., Bergvall, N., Östlin, G., Micheva, G., \& Leksell, M. 2006, ApJ, 650,812

Zasov, A. V., Khoperskov, A. V., \& Saburova, A. S. 2011, Astron. Lett., 37, 374 